\title{
Nonalcoholic Fatty Liver Disease
}

National Cancer Institute

\section{Source}

National Cancer Institute. Nonalcoholic Fatty Liver Disease. NCI Thesaurus. Code

C84444.

A term referring to fatty replacement of the hepatic parenchyma which is not related to alcohol use. 